\title{
Producción de semilla de cilantro (Coriandrum sativum L.) bajo la incidencia de malezas y Alternaria Nees
}

\section{Coriander (Coriandrum sativum L.) seed production under weed and Alternaria Nees incidence}

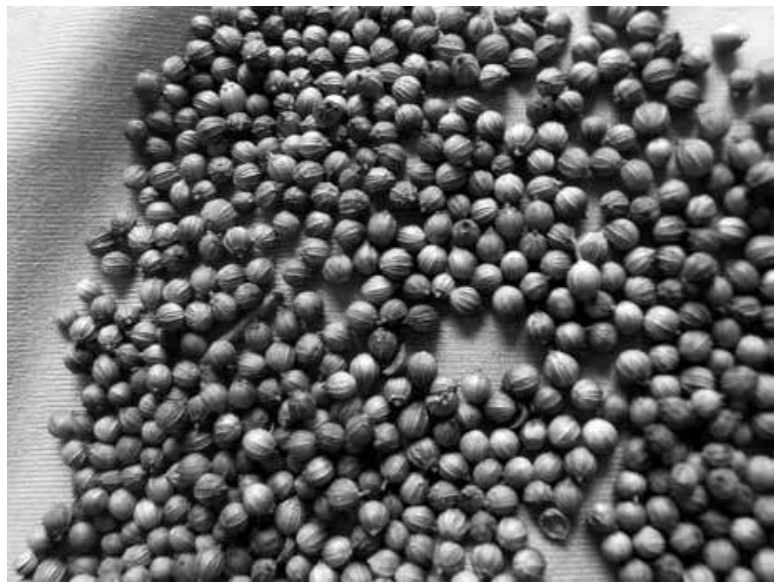

LAGUANDIO BANDA S. ${ }^{1,3}$

CILIA LEONOR FUENTES ${ }^{2}$

BERNARDO CHAVES ${ }^{2}$

\section{RESUMEN}

El daño causado por malezas, Alternaria e insectos plaga en la producción de semilla de cilantro fue el objeto de esta investigación. Se evaluaron atributos de la comunidad de malezas (especies, frecuencia, cobertura y densidad), incidencia y severidad de Alternaria, insectos plaga, masa seca y rendimiento en semilla de cilantro. Hubo diferencias significativas para las variables evaluadas entre intensidades (alto, medio, bajo y sin control) de manejo fitosanitario. La comunidad de malezas estuvo conformada por 15 especies; entre ellas, la de menor frecuencia fue Penisetum clandestinum (9\%) y la de mayor frecuencia (91\%) y densidad más alta (541 individuos $/ \mathrm{m}^{2}$ ) fue Lolium temulentum. Las densidades de malezas monocotiledóneas y dicotiledóneas fueron entre uno y cuatro plantas $/ \mathrm{m}^{2}$ a altas intensidades de manejo. Alternaria se presentó a los 50 días después de la siembra del cilantro y produjo daños severos al no controlarla, con valores mayores a un $65 \%$ de plantas infectadas y niveles de severidad con muerte total de plantas. Al implementar medidas de control intensas de Alternaria, los niveles de incidencia y severidad se aproximaron al 1,5\% (daños muy leves). La masa seca del cilantro se redujo $82 \%$ cuando no se controló Alternaria y $92 \%$ bajo el tratamiento sin control de las malezas, respecto a controles altos. El rendimiento de semillas de cilantro fue entre 167,1 y $174,3 \mathrm{~g} \mathrm{~m}^{-2}$ con la mínima presencia de malezas, de Alternaria y ausencia de insectos plaga. Sin control de malezas no se obtuvo semilla de cilantro, y cuando no se controló Alternaria la cantidad de semilla de cilantro obtenida fue de $7,5 \mathrm{~g} \mathrm{~m}^{-2}$.

1 Facultad de de Ciencias Agropecuarias, Programa de Ingeniería Agronómica, Universidad de Cundinamarca, Fusagasugá (Colombia).

2 Facultad de Agronomía, Departamento de Agronomía, Universidad Nacional de Colombia, Bogotá (Colombia).

3 Autor para correspondencia.laguandio@gmail.com, laguandio@yahoo.com 


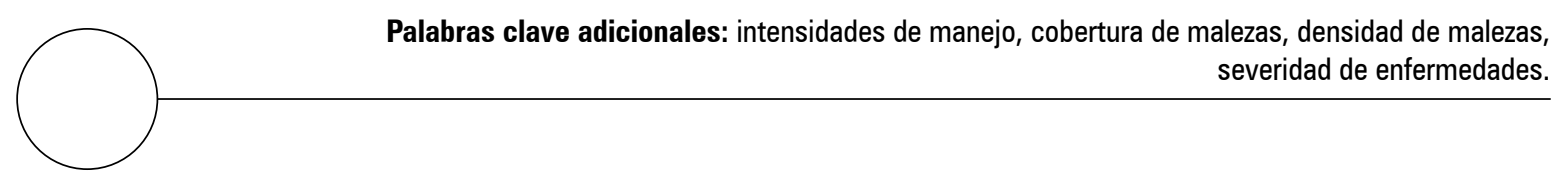

ABSTRACT

The damage caused by weeds, Alternaria fungus and insect pests on the production of coriander seed was studied. The present research evaluated the attributes of weeds (species, frequency, cover and density), incidence and severity of Alternaria, insect pest, dry matter and yield in coriander seed. There were significant differences for the evaluated variables between intensities (high, medium, low and no control) of weed and Alternaria management. The weed community was composed of 15 species: lowest frequency Penisetum clandestinum (9\%) and highest Lolium temulentum (91\%). L. temulentum had the highest density (541 individuals $/ \mathrm{m}^{2}$ ). The densities of monocotyledonous and dicotyledonous weed species with high control registered between one and four plants $/ \mathrm{m}^{2}$. Alternaria affected coriander at day 50 after sowing and produced severe damage when no control was taken; with $65 \%$ infected plants and a level of severity of total plant death. When intensive control measures of Alternaria were taken, the levels of incidence and severity stayed near $1.5 \%$ with very slight damage. Coriander dry matter had a reduction of $82 \%$ due to Alternaria without any control measures and $92 \%$ with no weed control, compared to the control. The production of coriander seeds was between 167.1 and $174.3 \mathrm{~g} \mathrm{~m}^{-2}$ with a minimal presence of weeds and Alternaria, and the absence of insect pests. No seed was obtained without weed control and without control of Alternaria, seed yield was $7.5 \mathrm{~g} \mathrm{~m}^{-2}$.

Additional keywords: management intensities, weed cover, weed density, disease severity.

Fecha de recepción: 29-01-2011

Aprobado para publicación: 21-11-2011
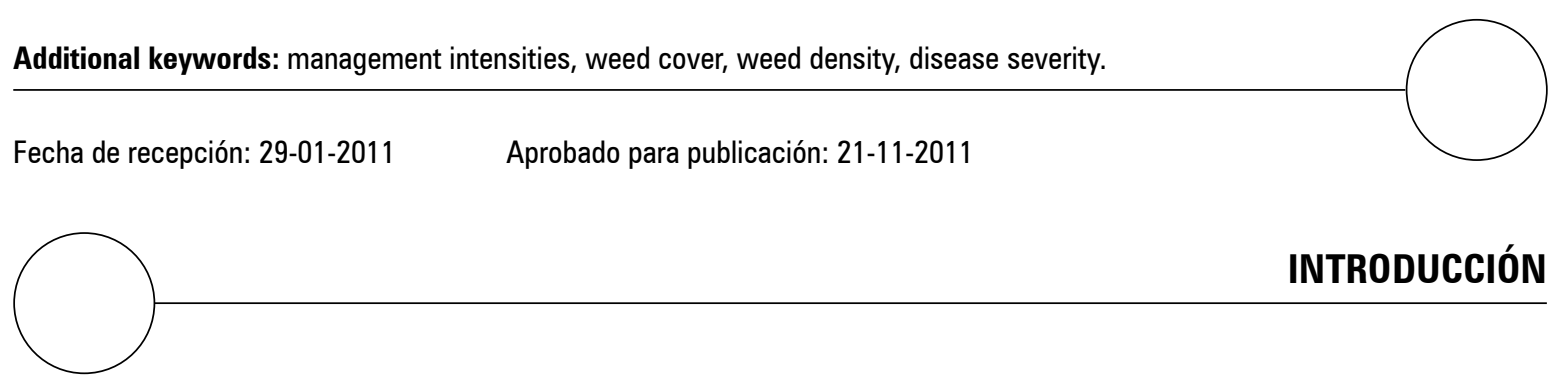

INTRODUCCIÓN

El cilantro (Coriandrum sativum L., Umbelliferae, Apiaceae) es una planta de uso culinario. En Colombia, la variedad Fino de Castilla o Patimorado, está adaptada entre los 800 y 2.800 msnm (Acuña, 1998; Estrada et al., 2004), y se siembran unas 1.962 ha de las 113.773 ha de hortalizas anualmente, donde Cundinamarca participa con el 30 \% de la producción (Ministerio de Agricultura y Desarrollo Rural, 2009).

En todo cultivo, el manejo preventivo de los problemas fitosanitarios incluye la utilización de material de propagación o semilla sana, libre de cualquier agente considerado como plaga (maleza, insecto o patógeno) (Besnier, 1989; Fuentes y Plaza, 1992; Ávila y Velandia, 1992; López, 1992). Se requiere entre 45 a 120 d para obtener semilla (Acuña, 1988; Diederichsen, 1996; Estrada et al., 2004).

Fuentes y Plaza (1992) registraron 32 especies de malezas como las más frecuentes en los cultivos hortícolas de la Sabana de Bogotá, de las familias: Poaceae, Fumariaceae, Chenopodiaceae, Cruciferae, Polygonaceae, Compositae, Amaranthaceae, Scrophulariaceae, Malvaceae, Cruciferae. Información fue reafirmada por Eraso y Sequeda (2005), quienes registraron 143 especies arvenses en el Altiplano Cundiboyacense.

Las malezas o arvenses afectan la producción del cilantro: disminuyen rendimientos y deterioran la calidad del follaje y semilla, por la competencia o efectos alelopáticos sobre los cultivos (Al- 
drich, 1984, Radosevich y Roush, 1990; Anaya et al., 1992; Cousens y Mortimer, 1995; Gallagher et al., 1999).

En Colombia se han reportado enfermedades del cilantro como marchitamiento y pudrición de plántulas por Fusarium sp., Pythium sp. y Rizoctonia sp., el añublo foliar y quemazón de hojas por Alternarelia sp., Cercospora sp. y Phytophthora sp. Bacterias: Xanthomonas sp. (Estrada et al., 2004). En la Sabana de Bogotá, Martínez (1998) reportó al tizón o alternariosis causada por Alternaria dauci (Kuehn.) Groves y Skolko, que afecta al follaje y se puede diseminar por las semillas. Buriticá (1999) reportó en Antioquia, Cundinamarca y Nariño, a Alternaria Nees como hongo fitopatógeno causante de la mancha foliar del cilantro o Alternaria. Estrada et al. (2004) referenciaron plagas del cilantro: Spodoptera sp. y Agrotis sp., Epitrix sp., Diabrotica sp. Mizus sp., Thrips sp. y Lyriomiza sp. Otra plaga es el áfido Rhopalosipum sp. (Acuña, 1988), mientras que en Brasil, Araujo (1986) relaciona al áfido Myzus persicae (Sulzer).

En esta investigación se propuso conocer la incidencia de las malezas (especies, frecuencia, densidad y cobertura), del hongo Alternaria incidencia y severidad y los insectos plaga, sobre la producción de semilla de cilantro, sometidos a diferentes niveles de manejo, evaluando los rendimientos del cilantro en materia seca y semilla, bajo condiciones experimentales (Sabana de Bogotá, Colombia), ya que es importante que se produzca localmente semilla de cilantro.

\section{MATERIALES Y MÉTODOS}

Se instaló un experimento de campo en el Centro Agropecuario Marengo durante el segundo semestre de 2004 ( $4^{\circ} 42^{\prime}$ latitud norte y 74 12' longitud oeste, meridiano de Greenwich) de la Universidad Nacional de Colombia, Mosquera (Cundinamarca); a una altura de $2.543 \mathrm{msnm}$, con promedios anuales de $13^{\circ} \mathrm{C}$ de temperatura, $78 \%$ de humedad relativa, alrededor de $7 \mathrm{~h} \mathrm{día}^{-1}$ de brillo solar y $665 \mathrm{~mm}$ de precipitación anual (CAM, 2004).

\section{Establecimiento del cultivo de cilantro}

Se sembró la variedad de cilantro nacional (cultivado en Colombia) Fino de Castilla (Patimorado). La densidad de siembra fue de $20 \mathrm{~kg}$ $h^{-1}$. Para el manejo de malezas dicotiledóneas se utilizó linuron en dosis de $0,45 \mathrm{~kg} \mathrm{ha}^{-1}$ i.a. y para gramíneas propaquizafop en dosis de 0,12 $\mathrm{kg} \mathrm{ha}^{-1}$ i.a. El manejo de Alternaria se realizó con mancozeb a $0,75 \mathrm{~kg} \mathrm{ha}{ }^{-1}$ i.a. (protectante), y carbendazim aplicando $0,15 \mathrm{~kg} \mathrm{ha}^{-1}$ i.a. (sistémico, preventivo - curativo). Para insectos plaga no fue necesario implementar medidas de control.

\section{Diseño experimental}

El diseño experimental fue de bloques completos al azar con parcelas divididas, doce tratamientos y cuatro repeticiones. Cada bloque conformado por tres parcelas principales, definidas por la presencia de malezas, Alternaria e insectos plaga en el cultivo de cilantro. Las subparcelas dadas por las intensidades de control o manejo (sin control, bajo, medio y alto) de los problemas sanitarios (tabla 1). El área de cada unidad experimental (UE) o subparcela (SP) fue de $32,4 \mathrm{~m}^{2}$. Para las malezas se definieron los niveles de control con base en la frecuencia de cada control. Se tomó como referencia a Hill y Santelman (1969). Para Alternaria, los niveles de control dados por la frecuencia de cada control, frecuencia definida según el ciclo de patogénesis, alrededor de $21 \mathrm{~d}$ (Rottem, 1995; Agrios, 2005).

\section{Evaluación de la comunidad de malezas}

Se determinaron las especies presentes en el área experimental y caracteres de frecuencia (presencia de cada especie de arvenses en las UE para los diferentes muestreos realizados); cobertura (porcentaje del área del suelo cubierto por las malezas, de 20 posibles puntos dentro del cuadrado [n/20]) y densidad (número de individuos de una 
Tabla 1. Establecimiento de tratamientos en el área experimental.

\begin{tabular}{|c|c|c|}
\hline Tratamiento & Abreviatura & Descripción \\
\hline T1 Malezas sin control & msc & Sin control durante el ciclo del cultivo de cilantro \\
\hline T2 Malezas control bajo & mcb & Dos controles: 60 y 120 días después de la siembra (dds) del cilantro \\
\hline T3 Malezas control medio & $\mathrm{mcm}$ & Controles: 30, 60, 90 y 120 dds del cilantro \\
\hline T4 Malezas control alto & mca & Controles: cada 20 dds del cilantro (cultivo entre un 95 y $100 \%$ libre de malezas) \\
\hline T5 Alternaria sin control & asc & Sin medidas de control \\
\hline T6 Alternaria control bajo & acb & Controles cada 21 días después de la aparición de la enfermedad (ddae) \\
\hline T7 Alternaria control medio & acm & Controles cada 14 ddae \\
\hline T8 Alternaria control alto & aca & Controles cada 7 ddae \\
\hline T9 Insectos plaga sin control & ipsc $^{*}$ & Sin medidas de control \\
\hline T10 Insectos plaga control bajo & ipcb* & Controles cada 21 días después de la aparición del insecto plaga (ddaip) \\
\hline T11 Insectos plaga control medio & ipcm* & Medidas de control cada 14 ddaip \\
\hline T12 Insectos plaga control alto & ipca* & Controles cada 7 ddaip \\
\hline
\end{tabular}

* Ipsc, Ipcb, Ipcm e Ipca = maca: malezas y Alternaria control alto.

especie de maleza que se registraron en una área de $275 \mathrm{~cm}^{2}$ ) de las arvenses. La metodología de evaluación utilizada fue la propuesta por Fuentes (1986), usando el método del "cuadrado". El área del cuadrado fue de $0,25 \mathrm{~m}^{2}$, tomando tres muestras por UE.

\section{Evaluación de Alternaria}

Para Alternaria se evaluó la incidencia (inal) y la severidad (seval). Según Nutter (1997) la incidencia definida como el número de unidades (plantas) de muestreo en los que se registró la enfermedad (porcentaje de plantas afectadas), y la severidad, como el área de la unidad de muestreo enferma (expresada en niveles de cero a nueve), a partir del porcentaje de área foliar de la planta de cilantro afectada por Alternaria. Se utilizó el cuadrado de la evaluación de malezas, con muestreos cada 7 d. Se definieron tres muestras por UE tomadas al azar de 15 puntos posibles de muestreos. Se tomaron 20 plantas para evaluar la incidencia y cinco plantas para evaluar la severidad de Alternaria. El sistema de evaluación de Alternaria se adaptó de Castaño (1989), definiendo nueve niveles de severidad: (0) ningún síntoma visible de la enfer- medad (0\% área foliar afectada), (1) los síntomas son insignificantes o leves $(\approx 1$ a $5 \%$ de área foliar afectada), (3) síntomas y daños leves ( $\approx 6$ al 20\% de área foliar afectada), (5) plantas que presentan síntomas de daño moderado ( $\approx 21$ al 40\% de área foliar afectada), (7) material muy enfermo $(\approx 41$ al $60 \%$ de área foliar afectada), (9) material muy enfermo e incluye plantas muertas (mayor del $60 \%$ área foliar afectada).

\section{Evaluación de insectos plaga}

No se presentaron insectos plaga de importancia económica, por lo cual no se incluyen datos de resultados.

\section{Masa seca de las plantas de cilantro}

Se evaluó la masa seca (masec) a los 120 dds, cuando se encontraba alrededor del $80 \%$ de frutos formados. La muestra consistió en la planta total de cilantro, con tres muestras por UE y área de $1.600 \mathrm{~cm}^{2}$. Cada muestra fue llevada al Laboratorio de Fisiología de la Facultad de Agronomía Universidad Nacional de Colombia, donde se secaron en una estufa a $80^{\circ} \mathrm{C}$ durante $48 \mathrm{~h}$. 


\section{Producción de semilla de cilantro}

Se tomaron tres muestras de la semilla (en $\mathrm{g} \mathrm{m}^{-2}$ ) de cilantro producida por UE, en un área de 7,2 $\mathrm{m}^{2}$ por UE.

\section{Análisis de la información}

Se planteó el contraste de problemas limitativos de malezas, el hongo Alternaria e insectos plaga, con diferentes niveles de manejo. El diseño experimental se ajustó a bloques completos al azar, con nueve tratamientos: T1 a T4 (diferentes niveles de manejo de malezas); T5 a T8 (diferentes niveles de manejo de Alternaria) y T9 (un testigo con manejo alto de malezas y Alternaria), T1 a T8 contaron con cuatro replicas y $\mathrm{T} 9$ con 16 repeticiones. Se realizó un análisis de varianza (Anova) $y$ contrastes ortogonales, con el fin de dar respuesta a preguntas específicas sobre el efecto de los tratamientos en cada una de las variables evaluadas La información se procesó con el programa Statistical Analisis System (SAS) versión 9.1.

\section{RESULTADOS Y DISCUSIÓN}

\section{Especies de malezas en el área experimental}

La comunidad de malezas que interactuó con el cultivo de cilantro durante el experimento, estuvo conformada por 15 especies de malezas de 10 familias (tabla 2), comunes en la Sabana de Bogotá (Eraso y Sequeda, 2005). La determinación de especies se realizó mediante la literatura e investigaciones relacionadas (Eraso y Sequeda, 2005; Fuentes et al., 2006; Bermúdez, 1997; Holm et al., 1997). De las 15 especies de malezas presentes en el experimento, se encuentran varias catalogadas como medianas a altamente perjudiciales o nocivas (Trujillo, 1981), dado por la alta competitividad, daño en el cultivo, costo y posibilidades de erradicación como lo son: Lolium temulentum (Lolte), Brassica rapa (Brara), Urtica urens (Urtur), Rumex crispus (Rumcr), y Chenopodium paniculatum (Chepa), consideradas malezas comunes (MC) y nocivas ( $\mathrm{MN}$ ) (Resolución 2228 del ICA, 1983). Todas las especies

Tabla 2. Especies de malezas en el área experimental.

\begin{tabular}{|c|c|c|c|c|c|}
\hline No. & Especie & Nombre común & Familia & Abreviatura & $\begin{array}{c}\text { Calificación } \\
\text { Resolución } 2228 \\
\text { (MC, MN) }{ }^{1} \text { (ICA } \\
1983)\end{array}$ \\
\hline \multicolumn{6}{|c|}{ Monocotiledóneas } \\
\hline 1 & Lolium temulentum $\mathrm{L}$. & Raygras & \multirow{3}{*}{ Poaceae } & Lolte & $\mathrm{MC}$ \\
\hline 2 & Avena fatua L. & Avena silvestre & & Avefa & MN \\
\hline 3 & Penissetum clandestinum Hochst & Kikuyo & & Pecla & MN \\
\hline \multicolumn{6}{|c|}{ Dicotiledóneas } \\
\hline 4 & Amaranthus hybridus L. & Amarantus, bledo & Amaranthaceae & Amahy & $\mathrm{MC}$ \\
\hline 5 & Brassica rapa L. & Nabo, alpiste & Brassicaceae & Brara & MN \\
\hline 6 & Chenopodium panicultaum Hook & Cenizo & Chenopodiaceae & Chepe & MC \\
\hline 7 & Fuertesismalva limensis (L.) Fryxell & Malva blanca & Malvaceae & Fueli & $\mathrm{MN}$ \\
\hline 8 & Polygonum seguetum H.B.K. & Gualola & \multirow{2}{*}{ Polygonaceae } & Polse & $\mathrm{MC}$ \\
\hline 9 & Rumex crispus L. & Lengua de vaca & & Rumcr & $\mathrm{MN}$ \\
\hline 10 & Sonchus oleraceus L. & Cerrajo & \multirow{3}{*}{ Asteraceae } & Sonol & $\mathrm{MC}$ \\
\hline 11 & Sonchus asper (L.) Hill & Cerrajo & & Sonas & $\mathrm{MC}$ \\
\hline 12 & Galinsoga ciliata (Raf) Blake & Guasca & & Galci & $\mathrm{MC}$ \\
\hline 13 & Urtica urens L. & Ortiga blanca & Urticaceae & Urtur & MN \\
\hline 14 & Fumaria capreolata L. & Fumaria & Fumariaceae & Fumca & - \\
\hline 15 & Veronica persica Poir & Golondrina & Scrophulariaceae & Verpe & - \\
\hline
\end{tabular}

${ }^{1}$ MN: maleza nociva; MC: maleza común. 
de malezas alcanzaron la fase reproductiva en el tratamiento malezas sin control (msc).

Frecuencia de las especies de malezas

Para la frecuencia de las especies de malezas en el área experimental (figura 1). Lolte se registró en un $91 \%$ de las muestras, seguido por frecuencias mayores de 39\%, para Fueli, Amahy, Brara, y Chepe (58\%, 48\%, 46\% y 39\%, respectivamente), mientras que la maleza de menor frecuencia fue Pecla con un $9 \%$ de frecuencia. La frecuencia de cada maleza en el experimento, puede ser una referencia de las que generaron mayor competencia al cilantro, especialmente en UE sin manejo, manejo bajo y medio de malezas.

\section{Densidad de las especies de malezas}

La densidad para las especies de malezas (figura 2) muestra que Lolte tuvo una densidad promedio de 541 tallos $/ \mathrm{m}^{2}$. Las dicotiledóneas con promedios de más de 50 individuos $/ \mathrm{m}^{2}$, fueron Amahy, Urtur, Fueli, Chepe, Verpe y Brara. Mientras que la menor densidad la presentó Galci, con ocho individuos $/ \mathrm{m}^{2}$. Valores que predominaron en $\mathrm{msc}$, mcb y $\mathrm{mcm}$.

La densidad de malezas monocotiledóneas (dmm) y dicotiledóneas ( $\mathrm{dmd}$ ) se representa en la figura 3. En msc se presentó la mayor dmd (943 plantas $/ \mathrm{m}^{2}$ ), mientras que en mcb se presentó la más alta dmm (980 plantas/m²). Los tratamientos en los que se implementó un nivel de control alto de malezas y Alternaria (mca, aca y maca), presentaron densidades de malezas similares, con valores menores a 25 plantas $/ \mathrm{m}^{2}$.

La dmm y dmd (tabla 3) registraron diferencias significativas entre tratamientos $(P<0,0001)$. Los contrastes ortogonales (tabla 4$)$ registraron para las dos variables, diferencias estadísticas entre los

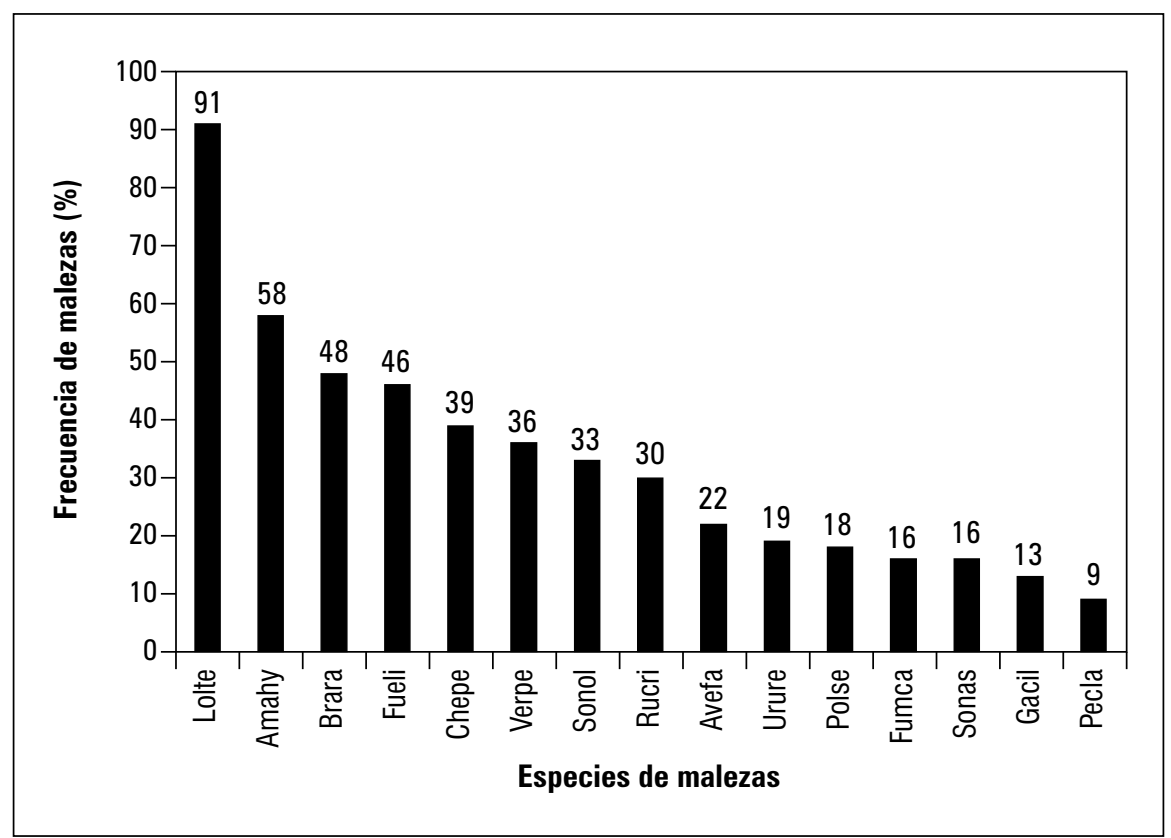

Figura 1. Frecuencia de las malezas en el área experimental con el cultivo de cilantro. Lolte (L. temulentum), Urtur (U. urens), Pecla (P. clandestinum), Avefa (A. fatua), Chepe (C. petiolare), Brara (B. rapa), Polse (P. segetum), Sonol (S. opleraceus), Fumca (F. capreolata), Fueli (F. limensis), Amahy (A. hybridus), Verpe (V. persica), Rumcr (R. crispus), Sonas (S. asper), Gacil (G. ciliata). 


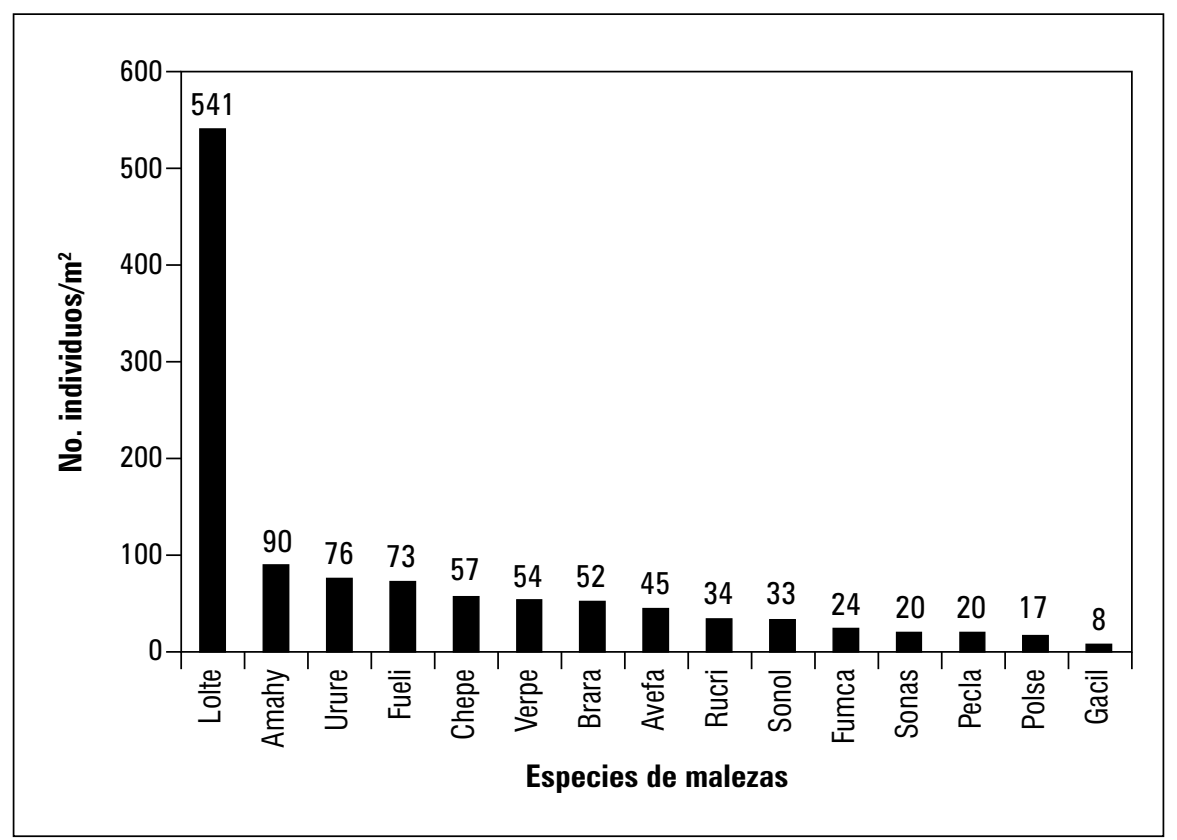

Figura 2. Densidad de las malezas en el área experimental con el cultivo de cilantro. Lolte (L. temulentum), Urtur (U. urens), Pecla (P. clandestinum), Avefa (A. fatua), Chepe (C. petiolare), Brara (B. rapa), Polse (P. segetum), Sonol ( $S$. opleraceus), Fumca (F. capreolata), Fueli (F. limensis), Amahy (A. hybridus), Verpe (V. persica), Rumcr (R. crispus), Sonas (S. asper), Gacil (G. ciliata).

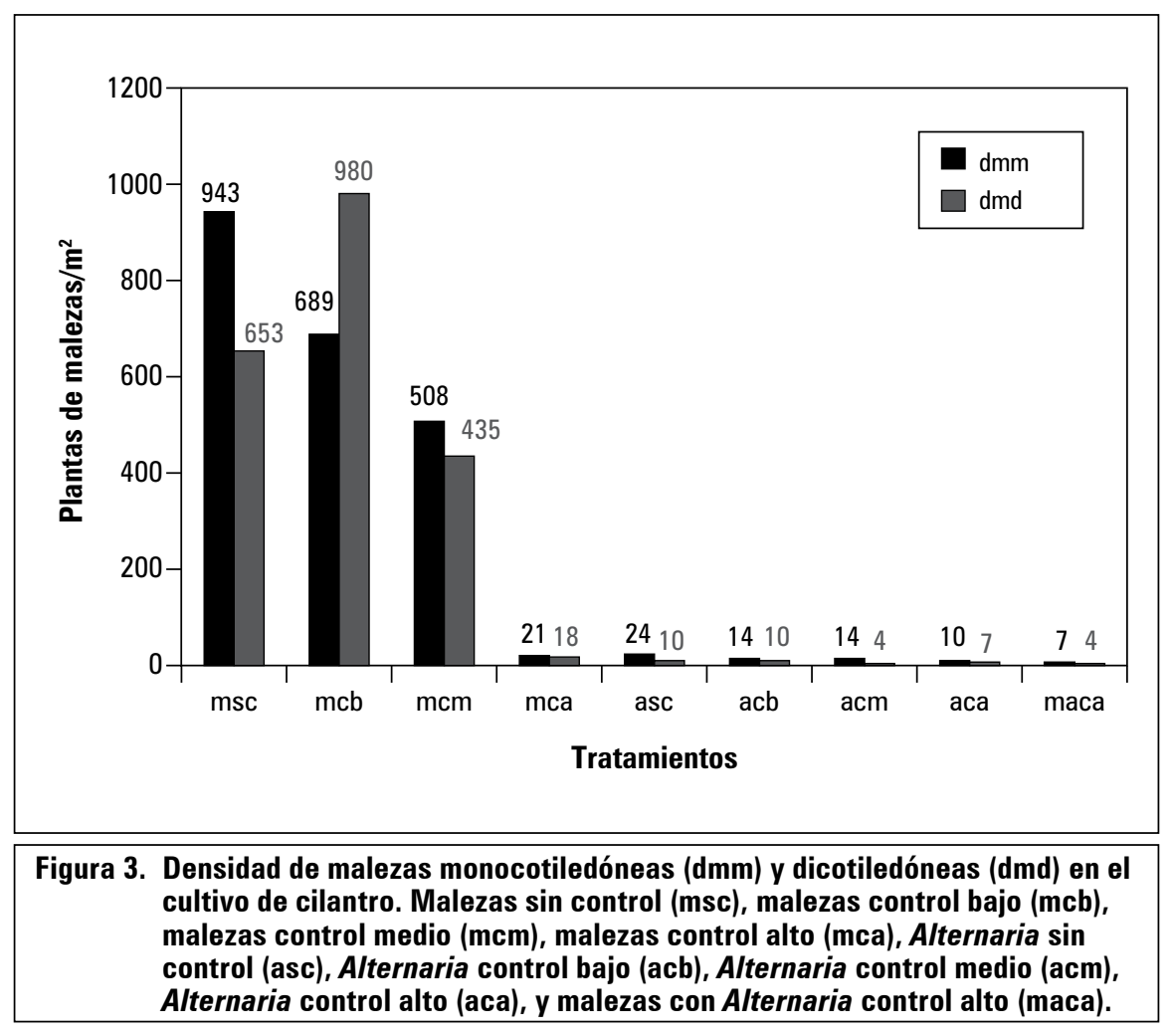


diferentes niveles de manejo de malezas (msc, mcb, mcm, mca), y entre maca con respecto a los manejos de malezas. Para maca con respecto al manejo de Alternaria (asc, acb, acm, aca), no fueron significativas las diferencias, esto por la implementación de un mismo nivel de control de malezas (control alto) en esos tratamientos.

\section{Cobertura de malezas}

La cobertura de malezas (comal) presentó diferencias significativas en el Anova $(P<0,0001)$ (tabla 3 ), donde alcanzó valores de $62,50 \%$ en msc, y del $3 \%$ en mca. En los tratamientos maca y de manejo de Alternaria, con controles altos de malezas, la cobertura se mantuvo alrededor de
$12 \%$, valores de cobertura obtenidos de las malezas que emergieron entre un control y otro, además, sumado a las que escapan a los controles implementados (figura 4).

Los contrastes ortogonales para comal (tabla 4) registraron diferencias significativas para los diferentes manejos de malezas: entre manejos de malezas y demás tratamientos, mas no se presentaron diferencias entre maca y manejos de Alternaria. Los resultados de los atributos (frecuencia, densidad y cobertura) evaluados para las especies de malezas, muestran a la comunidad de malezas como un factor muy limitativo del cultivo de cilantro.

Tabla 3. Significancia del Anova para las variables evaluadas en el experimento.

\begin{tabular}{|l|c|c|c|c|c|}
\hline Variable & F-valor & Pr $>F$ & $R^{2}$ & Coef. Var. & Media \\
\hline $\mathrm{dmm}$ & 19,30 & $<0,0001$ & 0,817 & 67,32 & 6,5625 \\
\hline $\mathrm{dmd}$ & 49,02 & $<0,0001$ & 0,917 & 50,50 & 6,6458 \\
\hline comal & 497,83 & $<0,0001$ & 0,991 & 18,83 & 0,1011 \\
\hline inal & 122,79 & $<0,0001$ & 0,964 & 40,20 & 0,0786 \\
\hline seval & 45,39 & $<0,0001$ & 0,990 & 18,12 & 0,1117 \\
\hline Masec & 323,88 & $<0,0001$ & 0,952 & 13,18 & 1,4854 \\
\hline Totsem & 217,80 & $<0,0001$ & 0,930 & 17,60 & 109,5570 \\
\hline
\end{tabular}

dmm, densidad de malezas monocotiledóneas; dmd, densidad de malezas dicotiledóneas; comal, cobertura de malezas; inal, incidencia de Alternaria; seval, severidad de Alternaria; Masec, peso masa seca; Totsem, producción total de semillas de cilantro.

Tabla 4. Significancia estadística de los contrastes ortogonales para las variables evaluadas en el experimento.

\begin{tabular}{|c|c|c|c|c|c|c|c|}
\hline \multirow{2}{*}{ Contraste } & \multicolumn{7}{|c|}{ Variables } \\
\hline & $\mathrm{dmm}$ & dmd & comal & inal & seval & Masec & Totsem \\
\hline Manejo de malezas vs. manejos Alternaria & $* *$ & $* *$ & $* *$ & $* *$ & $* *$ & $* *$ & ns \\
\hline Maca vs. resto & ** & ** & ** & ** & ** & ** & ** \\
\hline Maca vs. manejos malezas & ** & ** & ** & ns & ns & ** & $* *$ \\
\hline Maca vs. manejos Alternaria & ns & ns & ns & ** & ** & $* *$ & ** \\
\hline Msc vs. resto & $* *$ & ** & ** & ns & ns & ** & $* *$ \\
\hline Mcb vs. resto & $* *$ & ** & ** & ns & ns & $* *$ & ** \\
\hline Mcm vs. manejo malezas alto & $* *$ & $* *$ & $* *$ & ns & $\mathrm{ns}$ & $* *$ & $* *$ \\
\hline Asc vs. resto & ns & ns & ns & ** & ** & $* *$ & ** \\
\hline Acb vs. resto & ns & ns & ns & ** & $* *$ & $* *$ & $* *$ \\
\hline Acm vs. aca & ns & ns & ns & $* *$ & ** & $* *$ & ** \\
\hline
\end{tabular}

** Diferencias altamente significativas. ns: no significativo. 


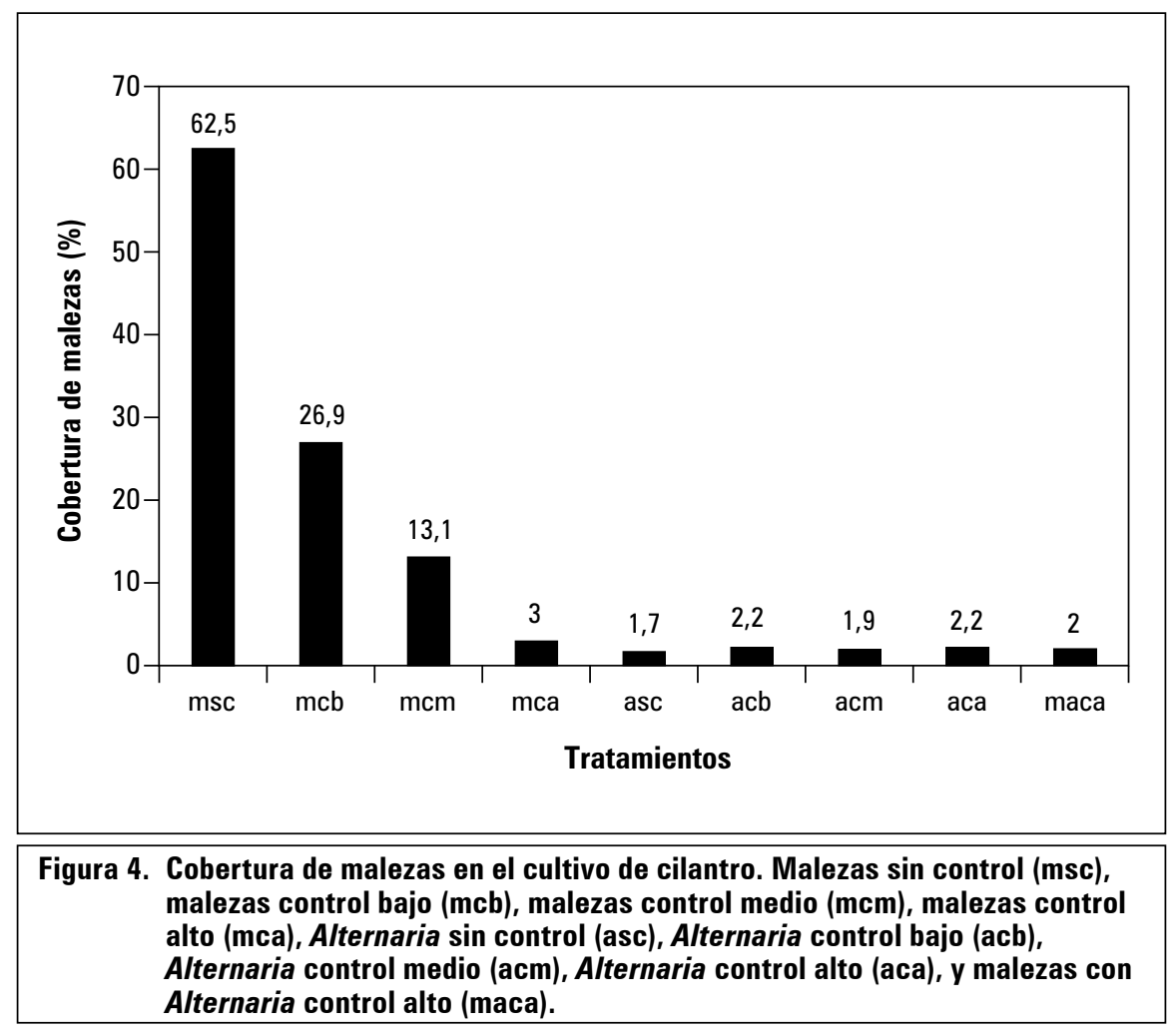

Las especies de malezas que compitieron con el cilantro fueron altamente agresivas. Aunque la distribución de todas las especies no fue homogénea, se resalta la alta frecuencia de Lolte, especie de alta competitividad con los cultivos. La densidad de las malezas varió entre 8 y 541 plantas $/ \mathrm{m}^{2}$. La densidad y cobertura de las malezas sirven como referencia del comportamiento y la interferencia de las especies arvenses con el cultivo de cilantro, y los posibles efectos en los rendimientos (Carlson y Hill, 1985).

El cilantro es muy sensible a la presencia de malezas durante el estado juvenil y de roseta (durante los primeros 30 a 40 d después de la emergencia), lo que implica la necesidad de un alto control de las mismas (Diederichsen, 1996; Estrada et al., 2004); en este caso, el cilantro se vio en desventaja con respecto a la comunidad de malezas para competir por los diferentes factores de crecimiento relacionados por Cousens y Mortimer (1995), especialmente cuando se disminuyeron los controles de las mismas.
La emergencia de todas las especies de malezas en el experimento fue al tiempo con el cultivo (con diferentes cohortes durante el ciclo del mismo), por lo que la competencia se dio desde el establecimiento del cultivo, aspecto determinante en los efectos de las malezas sobre los rendimientos de los cultivos (Dawson, 1971).

Las 15 especies de malezas que compitieron con el cultivo de cilantro y resaltando la toma de nutrimentos, se puede contrastar con lo encontrado en maíz (Zea mays L.) al competir con A. hybridus: alrededor del $28 \%$ de los contenidos nutricionales del suelo son aprovechados por la maleza (Vengris et al., 1955). Si esto sucede con A. hybridus, maleza presente en el área experimental, la competencia por nutrimentos pudo ser excesivamente alta en el caso del tratamiento msc. Se cita el caso evaluado por Cralle et al. (2003), donde el $L$. multiflorum mostró una alta competencia por el fósforo al interactuar con el cultivo de trigo, debido a un mejor aprovechamiento de nutrimentos a través de un mayor desarrollo radical. 
$\mathrm{Al}$ analizar la cobertura alcanzada por las malezas en los tratamientos $\mathrm{msc}$, mcb y mcm (figura 4), estas probablemente interfirieron sobre una buena cantidad y calidad de luz para el cultivo de cilantro. Estos efectos también pudieron darse a nivel de microambiente, sobre la disponibilidad de $\mathrm{CO}_{2}$, la disponibilidad de oxigeno en el área de influencia del las raíces, y efectos en las condiciones de temperatura del suelo y parte aérea de la planta. Elementos por los que también compiten o son determinantes del crecimiento de las diferentes especies de plantas (Evans y Young, 1972).

Las 15 especies de malezas que interactuaron con el cultivo de cilantro al presentar una alta densidad, cobertura y variada frecuencia, son aspectos que condicionan o limitan el manejo de la comunidad de malezas, a partir de los fundamentos de la época crítica de competencia y del concepto de umbrales.

Para la época o periodo crítico de competencia, que es aproximadamente equivalente al primer tercio o a la mitad del ciclo de vida del cultivo (Doll, 1999) o de mantener el cultivo libre de malezas en determinadas etapas de desarrollo (García y Fernández, 1991), y dada la variación del periodo crítico según las especies de arvenses (tres especies monocotiledóneas y 12 dicotiledóneas en la área experimental), la alta presión o competencia generada por las diferentes especies de malezas, como lo ocurrido en los tratamientos en los que se redujeron los niveles de control, especialmente msc, donde, los rendimientos del cilantro se redujeron a cero. Por tanto, se podría decir que las condiciones presentadas por la comunidad de malezas 'superan' o limitan la aplicabilidad del periodo crítico de competencia bajo las condiciones experimentales de campo manejadas.

Al igual que la poca aplicabilidad de la época crítica de competencia para el manejo de malezas en el experimento desarrollado, la aplicabilidad de umbrales también se ve limitada dada la complejidad de la competencia entre el cultivo de cilantro y las diferentes malezas que interactuaron con el mismo. Los conceptos de época crítica y de umbrales, generalmente, han sido evaluados y aplicados con especies de malezas a nivel individual y a cultivos muy específicos (Swanton et al., 1999), en este experimento la competencia fue dada por toda una comunidad de malezas cuya densidad, cobertura y distribución en la área experimental fue muy variada y compleja, por consiguiente, los niveles de la época critica de competencia y de umbrales se pudieron aproximar a niveles de cero.

\section{Incidencia y severidad de Alternaria en el cultivo de cilantro}

El cilantro presentó los primeros síntomas de Alternaria a los 50 dds. Estos síntomas se observaron primero en las hojas bajeras de las plantas (inicialmente pequeñas manchas foliares de color amarillento, que alrededor de los $5 \mathrm{~d}$ cambiaron a color café y luego variaron a color café oscuro y negro). Las manchas foliares de Alternaria empezaron a convertirse en quemazones del follaje alrededor de los $12 \mathrm{~d}$ después de la detección de los primeros síntomas. Estos síntomas observados de Alternaria en el cultivo del cilantro fueron similares a los descritos por Domínguez (1965), Torres (1995), Agrios (2005) y Estrada et al. (2004), característicos de este hongo fitopatógeno (Alternaria sp.).

Los valores más altos de la incidencia y severidad de Alternaria durante el ciclo del cultivo se registraron en asc $(65 \%$ de plantas y un nivel 6,4 en el follaje afectado) y en acb $(\approx 40 \%$ de plantas afectadas y un nivel de 5,2 de follaje afectado), mientras que en aca presentó valores próximos a $1 \%$ de las plantas afectas y nivel de severidad de 0,35 de follaje afectado (figura 5). Estas diferencias en el Anova fueron significativas $(P<0,0001)$ (tabla 3$)$.

Los contrastes ortogonales para la incidencia (inal) y severidad (seval) de Alternaria (tabla 4) 
muestran diferencias altamente significativas, así: entre los diferentes manejos de Alternaria, entre el manejo de Alternaria y el resto de tratamientos. No se presentaron diferencias significativas entre maca y los diferentes niveles de manejo de malezas. Alternaria produjo daños severos en el tratamiento sin control (asc), causando la muerte casi total de las plantas de cilantro en muchos casos. Este nivel de severidad fue alcanzado alrededor de los $60 \mathrm{~d}$ después de la aparición de la enfermedad.

Los tratamientos con los diferentes manejos de Alternaria, fueron determinantes en la presencia y desarrollo de la epidemia causada por este hongo fitopatógeno. En el caso de aca, los niveles de incidencia y severidad alcanzaron valores entre el $0 \%$ y $3 \%$ de plantas afectadas, mientras que en asc, hasta un $65 \%$ de plantas afectadas y severidad mayor a un nivel de 6 durante el ciclo del cultivo. Los daños en este último tratamiento llevaron a la muerte de las plantas.

\section{Masa seca del cilantro}

En la figura 6 se muestran los resultados de la masa seca (masec) de las plantas. Se muestra uno de los efectos de la competitividad de las malezas y el daño causado por Alternaria sobre el cultivo de cilantro. Los tratamientos mca, aca y maca presentaron los mayores valores de masa seca (2,1; 2,5 y $2,2 \mathrm{~g} /$ planta, respectivamente).

La masec registró diferencias altamente significativas $(P \leq 0,0001)$ (tabla 3$)$, igual los contrastes ortogonales (tabla 4). Diferencias entre los tratamientos con manejos de malezas (msc, mcb, $\mathrm{mcm}, \mathrm{mca}$ ) y de Alternaria (asc, acb, acm, aca), y entre estos grupos con respecto a los demás tratamientos.

La evaluación de masec fue una primera aproximación al grado de agresividad de las malezas y de Alternaria sobre el cultivo del cilantro. La masec se redujo entre un $92 \%$ y $82 \%$ en los tra-

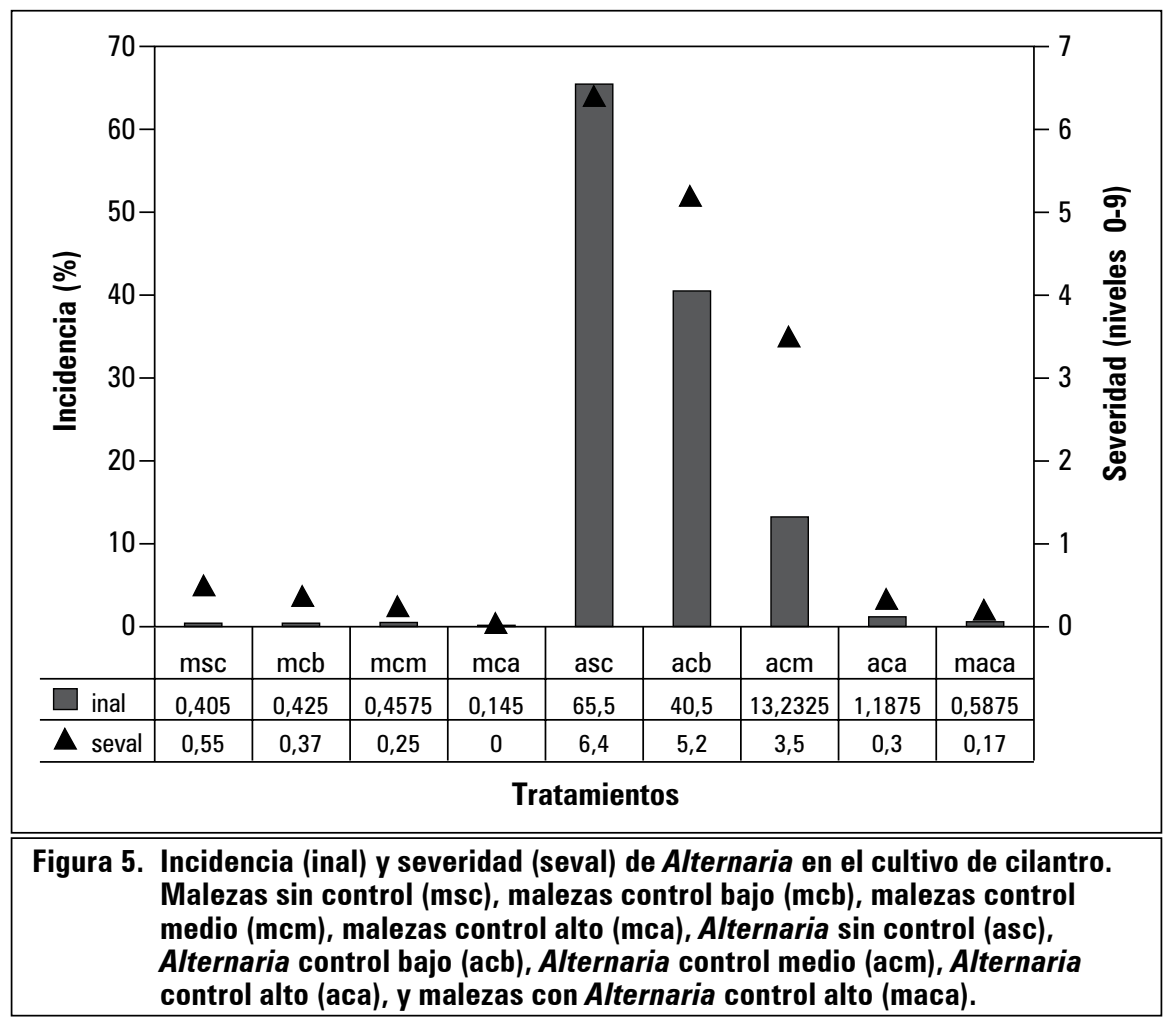

Vol. 5 - No. 2 - 2011 
tamientos sin control de malezas (msc) y de Alternaria (asc), con respecto a los controles altos (mca, aca, y maca) (figura 6). Esto es una muestra de la importancia del manejo de las malezas y Alternaria, que causan grandes perjuicios sobre el normal desarrollo de las plantas de cilantro en los tratamientos sin controles.

Al analizar los componentes esenciales de la materia seca de una planta: polisacáridos y lignina de la pared celular, componentes del protoplasma, incluyendo proteínas, lípidos, aminoácidos, ácidos orgánicos y determinados elementos como potasio (Salisbury y Ross, 1994), los cuales pudieron ser afectados por las malezas y Alternaria, quienes disminuyen los diferentes nutrimentos necesarios para un adecuado desarrollo de las plantas de cilantro. Daño indirecto causado por las malezas que compitieron con el cilantro por recursos del ambiente (agua, luz, nutrimentos, espacio) sin dejar de lado el posible efecto alelopático (Cousens y Mortimer, 1995) y un daño directo por Alternaria que obtienen los elementos esenciales de la planta de cilantro, con consecuencias en la producción de follaje y semilla.

\section{Rendimiento de semilla de cilantro}

La figura 7 muestra los valores de la producción de semillas de cilantro (Totsem) obtenida según los diferentes niveles de manejo de malezas y Alternaria. La mayor producción total fue alcanzada en el tratamiento mca con $174,26 \mathrm{~g} \mathrm{~m}^{-2}$, mientras que no se logró ninguna producción en msc. El Anova para la producción de semilla de cilantro, registró diferencias altamente significativas $(P \leq 0,0001)$ entre tratamientos (tabla 3 y 4$)$.

La pérdida total de la producción de semilla de cilantro en los tratamientos msc y en asc, muestran la importancia económica del control de las malezas y Alternaria para el cultivo de cilantro. La reducción de los rendimientos es notable en la medida que hay una mayor interferencia (com-

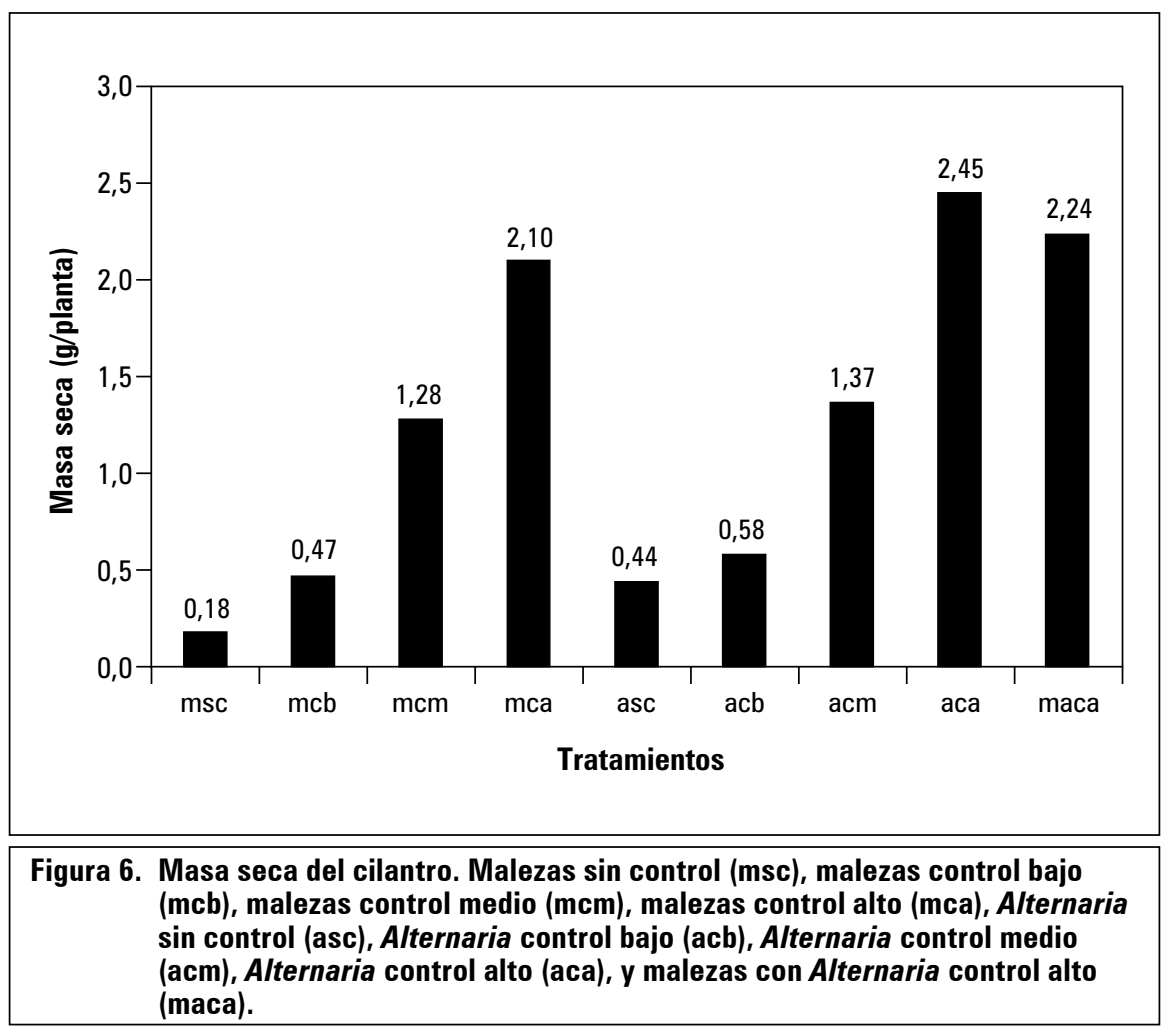

REV. COLOMB. CIENC. HORTIC. 
petencia) de las malezas con el cilantro, y similar resultado, cuando se disminuye la intensidad de control de Alternaria (figura 7).

Los bajos rendimientos de la semilla de cilantro, obtenidos en los tratamientos en los que se redujo el control de Alternaria, muestran la agresividad de este patógeno sobre el cilantro, teniendo en cuenta la agresividad mencionada por Neergaard (1977), quien relaciona a Alternaria como uno de los problemas que reduce los rendimientos en zanahoria entre un $5 \%$ y $55 \%$ bajo diferentes intensidades de control. Las pérdidas causadas por la comunidad de malezas sobre el cultivo de cilantro alcanzaron hasta un $94,7 \%$ del peso seco del cilantro con respecto al cultivo siempre limpio. Comparación que ratifica las pérdidas causada por la comunidad de malezas en la producción de semilla obtenida en esta investigación, similar a resultados obtenidos por Álvarez y Escandon (1990).
La competencia de la comunidad de malezas con el cilantro fue extremadamente alta, dada la reducción del $100 \%$ de la producción de semilla de cilantro en msc, y hasta en un $50 \%$ en mcm. Roberts et al. (1976) afirman que poblaciones de malezas con densidades entre 50 y 540 plantas/ $\mathrm{m}^{2}$, reducen los rendimientos en cultivos de crucíferas entre $47 \%$ y $100 \%$, en comparación cuando se tienen los cultivos libres de malezas. Caso que se reflejó en la producción de semilla de cilantro, a medida que se reducen los niveles de control de malezas.

La drástica reducción de la producción de semilla de cilantro, a causa de las malezas y Alternaria, demuestra la importancia del manejo de estos problemas sanitarios, ligados a las características biológicas de la planta de cilantro, al ser una planta herbácea poco competitiva con las malezas (Estrada et al., 2004), a lo que se le

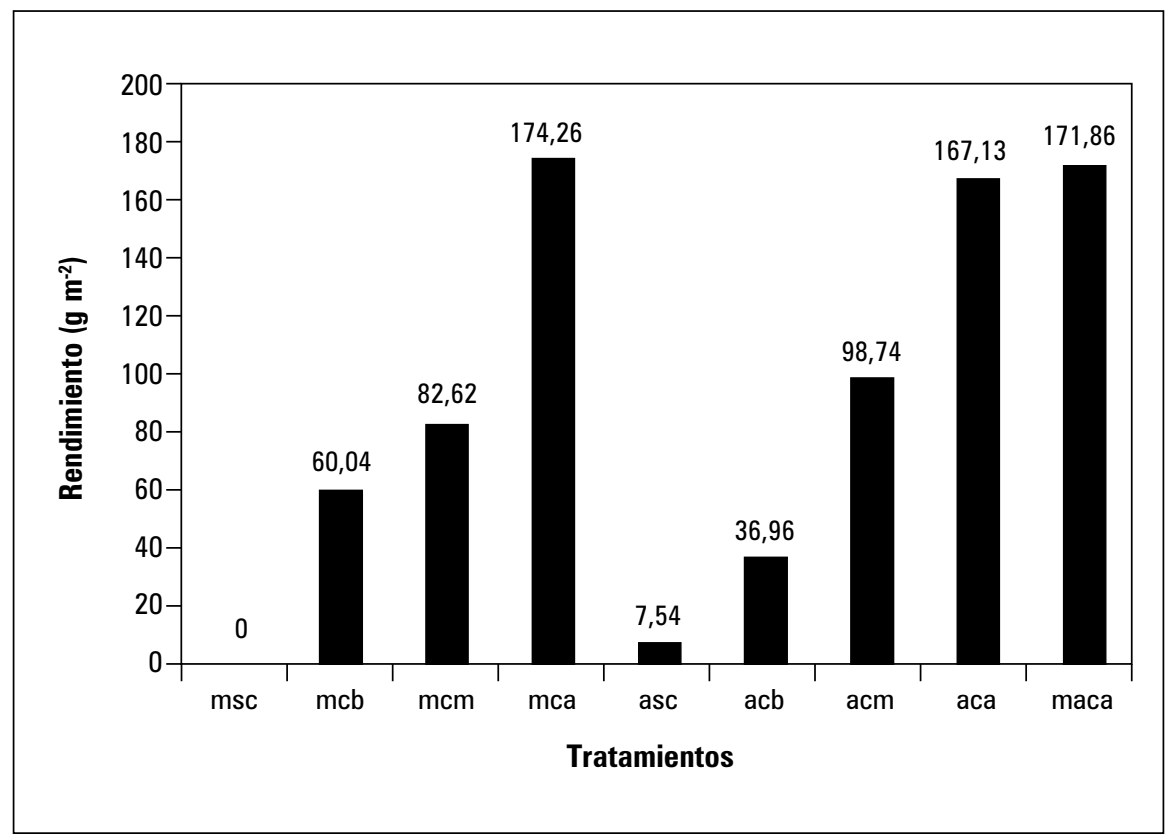

Figura 7. Rendimiento de semilla de cilantro. Malezas sin control (msc), malezas control bajo (mcb), malezas control medio $(\mathrm{mcm})$, malezas control alto (mca), Alternaria sin control (asc), Alternaria control bajo (acb), Alternaria control medio (acm), Alternaria control alto (aca), y malezas con Alternaria control alto (maca). 
suma la susceptibilidad del cilantro a Alternaria (Agrios, 2005; Neergaard, 1977), caso contrario a insectos plaga que no fueron de importancia económica.

\section{CONCLUSIONES}

Se evidenció la importancia de la presencia de las malezas y Alternaria sobre la producción de la semilla de cilantro, bajo diferentes niveles de manejo en la Sabana de Bogotá; en el caso de las plagas, no hubo presencia de insectos plaga de importancia económica. La comunidad de malezas que interactuó con el cilantro en el experimento desarrollado en la Sabana de Bogotá, Colombia, estuvo conformada por 15 especies, con predominio de Lolium temulentum, Brassica rapa, Fuertesismalva limensis, Chenopodium petiolare, Veronica persica y Rumex crispus, consideradas malezas altamente competitivas y agresivas en los diferentes sistemas de cultivos.

El grado de importancia de las especies de malezas se reflejó en la densidad promedio alcanzada en las unidades experimentales sin ningún control (msc) y con controles bajos (mcb), donde especies monocotiledóneas alcanzaron densidades hasta de 943 plantas $/ \mathrm{m}^{2}$, y especies dicotiledóneas 980 plantas $/ \mathrm{m}^{2}$, mientras que en los tratamientos con controles alto (mca, aca, maca) la densidad entre 4 y 24 plantas $/ \mathrm{m}^{2}$. Algo similar registró la cobertura, que alcanzó en msc hasta un $62,5 \%$ y en los tratamientos de control alto varió entre 1,7 y el $2,0 \%$.

La presencia de Alternaria en el cilantro ocurrió a los $50 \mathrm{~d}$ después de la siembra del cultivo. La in- cidencia de esta enfermedad alcanzó valores por encima del $65 \%$ en el tratamiento Alternaria $\sin$ control (asc) y mayores al 40\% en Alternaria control bajo (acb). El nivel de severidad que se registró estuvo por encima de 6 en asc y mayor a 5 en acb, esto representó al final del ciclo del cultivo plantas muy enfermas con muerte total en muchos casos. A medida que se intensificaron los controles de Alternaria se redujo el nivel de incidencia y severidad con valores cercanos a cero.

La evaluación de la materia seca del cilantro registró valores con reducciones drásticas entre un $82 \%$ y $92 \%$ en asc y msc, con respecto a los controles altos. Estos valores reflejan el alto grado de interferencia (competencia) de las malezas con el cultivo de cilantro y el daño directo causado por Alternaria sobre este cultivo.

Los máximos rendimientos en semilla de cilantro fueron alrededor de $170 \mathrm{~g} \mathrm{~m}^{-2}$, obtenidos en los manejos altos de malezas y Alternaria sin la presencia de insectos plaga; y cuando no se realizan manejo de malezas y Alternaria durante el desarrollo del cultivo de cilantro, los rendimientos en semilla se reduce en un 100\% y $95 \%$ respectivamente, respecto a controles altos.

\section{AGRADECIMIENTOS}

A la Facultad de Agronomía y al Centro Agropecuario Marengo de la Universidad Nacional de Colombia y a productores de semillas seleccionadas y a los señores Gómez y Méndez, productores de semillas seleccionadas, por la financiación de este estudio. 


\section{REFERENCIAS BIBLIOGRÁFICAS}

Acuña, R. 1988. Guía para la producción de hortalizas. Instituto Colombiano Agropecuario (ICA) y Secretaría de Agricultura, Cali, Colombia.

Agrios, G.N. 2005. Plant pathology. $5^{\text {a }}$ ed. Elsevier Academic Press, Burlington, MA.

Anaya, A.L., R.C. Ortega y R.V. Nava. 1992. Impact of allelopathy in the traditional management of agroecosystems in Mexico. pp. 271-301. En: Rizvi, S.J.H. y V. Rizvi (eds.). Allelopathy: Basic and applied aspects. Chapman \& Hall, London, UK.

Álvarez, S.J. y G.S. Escandon. 1990. Evaluación del control químico de malezas en el cultivo del cilantro (Coriandrum sativum L.) Trabajo de grado. Facultad de Ciencias Agropecuarias, Universidad Nacional de Colombia, Palmira.

Araujo, C.D. 1986. Occurrence of Myzus persicae (Sulzer, 1776) (Homoptera, Aphididae) on a crop of coriander Coriandrum sativum (Umbelliferae). Anais Soc. Entomol. Bras. 15, 173-174.

Ávila, C. y J. Velandia. 1992. Enfermedades de algunas especies hortícolas y su manejo. pp. 95-105. Primer Curso Nacional de Hortalizas de Clima Frió. ICA, Tibaitatá, Mosquera, Colombia.

Bermúdez, L.A. 1997. Malezas más comunes en Colombia. Produmedios, Bogotá.

Besnier, R.F. 1989. Semillas, biología y tecnología. Ediciones Mundi-Prensa. Madrid.

Buriticá, C.P. 1999. Directorio patógenos y enfermedades de las plantas de importancia económica en Colombia. Instituto Colombiano Agropecuario (ICA), Bogotá.

Carlson, H.L. y J.E. Hill. 1985. Wild oat (Avena fatua) competition with spring wheat: plant density effects. Weed Sci. 33, 176-181.

Castaño J. 1989. Estandarización de la estimación de daños causado por hongos bacterias y nematodos en fríjol (Phaseolus vulgaris L.). Fitopatol. Colomb. 13(1), 9-12.

Centro Agropecuario Marengo (CAM). 2004. Plan estratégico de desarrollo 2004-2008. Universidad Nacional de Colombia, Bogotá.

Cousens, R. y M. Mortimer. 1995. Dynamic of weed populations. University Press, Cambridge.
Cralle, H.T., T. Fojtasek, K. Carson, J. Chandler, T. Miller, S. Senseman, R. Bovery y M. Stone. 2003. Wheat and italian ryegrass (Lolium multiflorum) competition as affected by phosphorus nutrition. Weed Sci. 51, 425-429.

Dawson, J.H. 1971. Measuring effect of weeds on crops with emphas on weed-crop. Southern Weed Sci. Soc. 23, 13-25.

Diederichsen, A. 1996. Coriander (Coriandrum sativum L.). pp. 61-65. En: Promoting the conservation and use of underutilized and neglected crops. Institute of Plant Genetics and Crop Plant Research, Gatersleben. International Plant Genetic Resources Institute, Roma.

Doll, J.D. 1999. Dinámica y complejidad de la competencia de la maleza. pp. 35-49. En: Labrada, R., J. Casely y J. Parker (eds.). Manejo de malezas para países en desarrollo. FAO, Roma.

Domínguez, G.F. 1965. Plagas y enfermedades de las plantas cultivadas. $3^{\mathrm{a}}$ ed. Editorial Dossat, Madrid.

Eraso, C.E. y M.O. Sequeda. 2005. Contribución al reconocimiento de la flora arvense del altiplano cundíboyacense de Colombia. Trabajo de grado. Facultad de Agronomía, Universidad Nacional de Colombia, Bogotá.

Estrada, E., M. García, C. Cardozo, A. Gutiérrez, D. Baena, M. Salvador y F. Vallejo. 2004. Cultivo de cilantro variedad UNAPAL Precoso, Programa de investigación de hortalizas. Universidad Nacional de Colombia, Palmira, Colombia.

Evans, R. A. y J.A. Young. 1972. Microsite requirements for establishment of annual range land weeds. Weed Sci. 20, 350-356.

Fuentes, C.L. 1986. Metodología y técnicas para evaluar las poblaciones de malezas y su efecto en los cultivos. Revista Comalfi 13, 29-50.

Fuentes, C.L., A. Fúquene, E.M. Perdomo y S.C. Pinto. 2006. Plántulas de especies arvenses frecuentes en la zona centro de Colombia. Facultad de Agronomía, Universidad Nacional de Colombia, Bogotá.

Fuentes, C.L. y G. Plaza. 1992. Una aproximación al manejo de malezas en cultivos hortícolas de clima frío en Colombia. pp. 153-174. En: Primer Curso Nacional de Hortalizas de Clima Frío. ICA, Tibaitata, Mosquera, Colombia. 
Gallagher, R.S., E.C. Fernández y E.L. McCalle. 1999. Weed, management, through short term improved fallowa in tropical agroecosytems. Agrofor. Syst. 47, 197-221.

García, T.L. y Q.C. Fernández. 1991. Fundamentos sobre malas hierbas y herbicidas. Ministerio de Agricultura, Pesca y Alimento. Ediciones Mundi-Prensa, Madrid.

Hill, L.V. y P.W. Santelman. 1969. Competitive effects of annual weeds on Spanish peanuts. Weed Sci. 17, 1-2

Holm, L., J. Doll, E. Holm, J. Pancho y J. Herberger. 1997. World weeds: Natural histories and distribution. John Wiley \& Sons, New York, N.Y.

López, A. 1992. Plagas de las hortalizas y su manejo. pp. 117-152. En: Primer Curso Nacional de Hortalizas de Clima Frió. ICA, Tibaitata, Mosquera, Colombia.

Martínez, R.P. 1998. Enfermedades de las hortalizas en la Sabana de Bogotá y manejo fitosanitario general. ICA, División de Sanidad Vegetal, Bogotá.

Ministerio de Agricultura y Desarrollo Rural. 2009. Anuario estadístico de frutas y hortalizas 20042008. Bogotá. pp. 214-216.

Neergaard, P. 1977. Seed pathology. Vol. I. The Macmillan Press Ltd., New York, NY.

Nutter, F.W.J. 1997. Disease severity assessment training. pp. 1-7. En: Franci, L.J. y D.A. Neher (eds.). Exercises in plant disease epidemiology. APS Press, St. Paul, MN.
Radosevich, S.R. y M.L. Roush. 1990. The role of competition in agriculture. pp. 341-363. En: Grace, J. B. and D. Tilman (eds.). Perspectives on plant competition. Academic Press, New York, NY.

Roberts, H.A., W. Bond y R.T. Hewson. 1976. Weed competition in drilled summer cabbage. Ann. Appl. Biol. 84, 91-95.

Rottem, J. 1995. The genus Alternaria: biology, epidemiology and pathogenicity. APS Press, St. Paul, MN. pp. 326-329.

Salisbury, F.B. y C.W. Ross. 1994. Fisiología vegetal. Grupo Editorial Iberoamérica, México D.F.

Swanton, C.J., S. Weaver, P. Cowan, R. Van Acker, W. Deen y R. Shreshta, 1999. Weed thresholds: Theory and applicability. pp. 9-29. En: Buhler, D.D. (ed.). Expanding the context of weed management. Food Product Press, New York, NY.

Torres, G.C. 1995. Evaluación y diagnóstico de patógenos transmitidos por semillas en el cultivo de cilantro (Coriandrum sativum L.). Trabajo de grado. Facultad de Ciencias Agropecuarias, Universidad Nacional de Colombia, Palmira, Colombia.

Trujillo, B. 1981. Ecología de malezas. pp. 13-49. En: I Jornada técnica de especialistas en control de malezas. Sociedad Venezolana de Control de Malezas, Maracay, Venezuela.

Vengris, J., W.G. Colby y M. Drake. 1955. Plant nutrient competition between weed and corn. Agron. J. 47, 213-216. 